Relations industrielles

Industrial Relations

\title{
L'organisation du temps de travail dans les pays industrialisés, Genève, Bureau international du travail, 134 pp.
}

\section{René Boulard}

Volume 33, numéro 3, 1978

URI : https://id.erudit.org/iderudit/028899ar

DOI : https://doi.org/10.7202/028899ar

Aller au sommaire du numéro

Éditeur(s)

Département des relations industrielles de l'Université Laval

ISSN

0034-379X (imprimé)

1703-8138 (numérique)

Découvrir la revue

Citer ce compte rendu

Boulard, R. (1978). Compte rendu de [L'organisation du temps de travail dans les pays industrialisés, Genève, Bureau international du travail, 134 pp.] Relations industrielles / Industrial Relations, 33(3), 569-570.

https://doi.org/10.7202/028899ar

Tous droits réservés @ C Département des relations industrielles de l'Universite Laval, 1978
Ce document est protégé par la loi sur le droit d'auteur. L'utilisation des services d'Érudit (y compris la reproduction) est assujettie à sa politique d'utilisation que vous pouvez consulter en ligne.

https://apropos.erudit.org/fr/usagers/politique-dutilisation/ 
non grève, une deuxième qui exprime la durée de la grève en nombre de jours ouvrables. Il est intéressant d'apprendre que "l'activité de grève n'a pas contribué à l'accélération des salaires de l'ensemble des principales conventions collectives du secteur privé, ni à travers la détérioration du climat des relations industrielles, ni en occasionnant des augmentations de salaire supérieures pour les unités de négociation qui ont eu recours à la grève. L'activité de grève ne semble donc pas inflationniste " (p. 112).

Quant aux interventions dans le cadre des négociations collectives, il semble qu'elles n'entrainent pas de différences dans les ententes salariales. Il s'agit, tout comme la grève d'ailleurs, de mécanismes de transmission de l'information.

La thèse des auteurs est donc que dans le secteur privé le marché est déterminant; la négociation et éventuellement la grève sont des processus de transmission de l'information. Le secteur public est bien plus difficile à qualifier; en tout cas, il risque de générer des effets pervers dans son intéraction avec le secteur privé en aggravant des conséquences nationales des perturbations économiques mondiales. Le premier but d'une politique salariale doit donc être d'éviter l'apparition de disparités importantes entre les deux secteurs.

Au total, il s'agit d'un texte instructif et stimulant dans son orthodoxie.

Paul-Martel ROY

Université du Québec à Montréal

L'organisation du temps de travail dans les pays industrialisés, Genève, Bureau international du travail, $134 \mathrm{pp}$.

Le présent volume est le fruit d'un colloque sur l'aménagement du temps de travail et les problèmes sociaux relatifs au travail par équipes dans les pays industrialisés, tenu à Genève du 3 au 11 mai 1977. Il est composé de trois parties: la première présente deux documents de travail préparés par le BIT pour les participants du colloque; la deuxième reproduit le résumé de six des treize communications présentées au colloque; la dernière est constituée par le compte rendu des travaux du colloque.
Le premier document de travail fait le point sur les nouvelles tendances dans le domaine de l'aménagement du temps de travail. Ces tendances semblent marquées de deux caractéristiques principales: 1 . les aspects qualitatifs ont pris le pas sur les préoccupations quantitatives (v.g. le choix individuel des horaires s'est substitué aux revendications pour réduire le nombre d'heures de travail); 2 . l'émergence d'une tendance à considérer la durée du travail dans des cadres toujours plus larges (v.g. les concepts de durée annuelle du travail, de durée de la vie active sont en train de se substituer à ceux de la semaine et de la journée de travail). Dans ce contexte, le document présente une excellente typologie des différentes formes d'aménagement et de réaménagement des temps de travail.

Le deuxième document, après avoir rappelé brièvement les principes d'organisation et les problèmes généraux du travail par équipes, dresse également une typologie intéressante des différents problèmes sociaux reliés au travail par équipes. Le document tente de tracer le portrait du travail par équipes dans le monde. C'est ainsi que nous apprenons que «selon les pays, de 15 à 30 pour cent des effectifs de la population active «travaillent par équipes» et «qu'en moyenne, on peut estimer que la moitié des travailleurs par équipes sont impliqués dans les systèmes à trois postes, comprenant donc un poste de nuit ». L'examen des problèmes sociaux touche à tous les aspects, depuis le coût humain et social du travail par équipes (santé, vie familiale, vie sociale) jusqu'à l'emploi féminin.

La seconde partie du volume, où sont présentés six documents de travail préparés par les participants, tente de faire le point sur la situation de l'organisation du temps de travail dans quatre pays (Australie, Canada, Royaume-Uni, Pays-Bas), en plus de rapporter deux contributions originales: la première sur les conséquences administratives et sociales du travail par équipes, la seconde sur la recherche d'un seuil d'utilisation optimale du travail par équipes (selon l'auteur, ce seuil se situerait à deux postes quotidiens; ce qui signifie disparition du poste de nuit).

La dernière partie rapporte brièvement le résultat des travaux du colloque. Tous 
les points abordés par les participants (journée de travail continue, aménagement de la semaine de travail, étalement des horaires de travail, horaires variables ou libres, travail à temps partiel, aménagement de l'année, aménagement de la vie de travail et problèmes sociaux du travail par équipe) sont repris de façon synthétique.

Les remarques à faire sur cet ouvrage sont les suivantes:

1. il s'agit de loin du meilleur ouvrage de synthèse sur l'organisation et les nouvelles formes d'aménagement des temps de travail.

2. Tous les aspects sont touchés, les avantages et inconvénients clairement identifiés, les conséquences de tous ordres présentées.

3. Les affirmations des participants ne sont jamais, dans tout le volume, étayées par des preuves issues de la recherche (une des dernières recommandations porte précisément sur la nécessité de poursuivre des travaux en ce sens), mais la qualité des participants et de leurs interventions en garantit vraisemblablement la validité.

4. Les tentatives, assez éparses, de voir les nouvelles formes d'organisation du travail (horaires variables, semaine comprimée, etc.) comme une solution aux problèmes du travail par équipes sont peu convaincantes. Les participants eux-mêmes ont noté que les solutions à ce problème devraient être davantage "individualisées" (i.e. examinées à la pièce) et faire preuve d'originalité dans la plupart des cas.

Au total, l'ouvrage du BIT se présente comme une pièce-maîtresse, un tour d'horizon complet et intelligent pour tous ceux que l'organisation des temps de travail intéresse.

René BOULARD

Université Laval

International Collective Bargaining : an Analysis and Case Study for Europe, by Jacques Rojot, Deventer (Nederland), Kluwer, 1978.

Le but de cet ouvrage est d'examiner les perspectives d'une négociation collective internationale dans le cadre théorique existant des relations industrielles.
Dans un premier chapitre, consacré aux systèmes de relations industrielles et la théorie de la négociation collective, Rojot se propose de bâtir un modèle de négociation collective adapté à l'étude ultérieure au plan international. Dans le deuxième chapitre, il traite de la Communauté économique européenne en tant que système de relations industrielles internationales, dans le troisième chapitre de la négociation collective internationale dans le cadre de la CEE, pour aboutir, dans le quatrième et dernier chapitre, à une étude de cas sur la société multinationale GlaverbelMécaniver (groupe B.S.N.).

L'approche systémique, inspirée de Dunlop, traduit une interprétation originale, en substituant, notamment, un élément d'intérêt commun, d'adaptation mutuelle (de «coopération antagoniste» selon Dubin, d' "antagonisme incomplet », «décision interdépendante» ou "precarious partnership », selon Schelling), plus opérationnel et moins émotionnel dit Rojot, à l'élément idéologique mis en relief par le professeur de Harvard, qu'il considère comme trop restrictif. Cet élément lui semble nécessaire à l'existence d'un système de relations industrielles conçu dans un monde moderne, celui que nous vivons, où chaque acteur est conscient non seulement de son propre rôle mais également de ceux des autres partenaires sociaux.

Pour Rojot, la négociation collective n'est pas l'unique processus du système de relations industrielles. Il coexiste avec d'autres, tels l'action à travers les partis politiques. D'autre part, le concept de négociation collective ne lui semble pas devoir être limité ni à la négociation d'un accord écrit ni à l'administration du contrat, nombre d'activités qui traduisent l'interaction des acteurs sociaux pouvant $y$ être incluses. Ce qui va dans le plein sens des tendances du Bureau international du Travail et, particulièrement, de Johannes Schregle.

L'auteur traite, à plusieurs reprises, de la négociation collective en tant que «joint regulation», en la confrontant, lorsqu'il aborde les problèmes européens, à la participation (de type coopératif), à l'autogestion et au «co-management», à la régulation par le gouvernement et, enfin, à la décision unilatérale de l'employeur. 